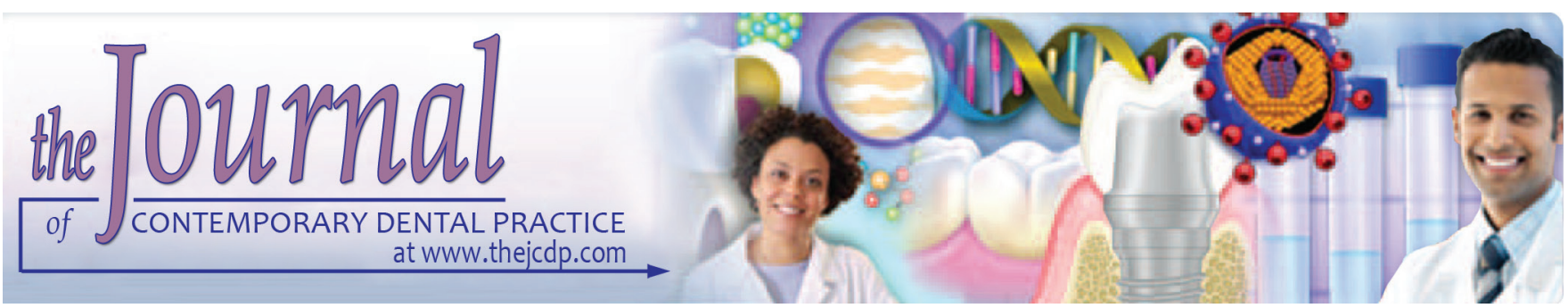

\title{
Study to assess Activity and Concentration of Gamma- glutamyl Transpeptidase in Precancerous and Cancer Patients and Its Comparison with Controls
}

\footnotetext{
${ }^{1}$ Anjum AJ Farooqui, ${ }^{2}$ Sanjay G Thete, ${ }^{3}$ Sayeedalaeque Bangi, ${ }^{4}$ Yogita J Khalekar, ${ }^{5}$ Neha D Nikumbh

${ }^{6}$ Keeran B Jadhav, ${ }^{7}$ Mitesh D Kathariya
}

\begin{abstract}
Objective: The purpose of the study is to highlight the use of gamma-glutamyl transpeptidase (GGT) as salivary enzyme tumor marker and assess the activity and concentration of GGT in precancerous and cancer patients and compare it with the normal control.
\end{abstract}

Materials and methods: Individuals in the age group of above 20 years were included in the study. In this study, salivary GGT was analyzed in 75 cases. The selected patients were divided into three main groups as group I (controls with normal health), group II (patients with precancerous lesions and conditions), and group III (patients with oral cancer lesions). All the selected individuals were analyzed for salivary GGT.

Results: A significant difference was observed between control and precancerous groups with GGT values at $5 \%$ level of significance. The mean GGT value is significantly higher in precancerous group as compared with control group $(p<0.05)$. A significant difference was observed between control and cancerous groups with GGT values at $5 \%$ level of significance. The mean GGT value is significantly higher in cancerous group as

\footnotetext{
${ }^{1,5,6}$ Department of Oral Medicine and Radiology, Rural Dental College, Pravara Institute of Medical Sciences, Loni Maharashtra, India

${ }^{2}$ Department of Oral Pathology, Rural Dental College, Pravara Institute of Medical Sciences, Loni, Maharashtra, India

${ }^{3}$ Department of Orthodontics, Al-Badar Rural Dental College \& Hospital, Gulbarga, Karnataka, India

${ }^{4}$ Department of Oral Medicine and Radiology, A.C.P.M. Dental College, Dhule, Maharashtra, India

${ }^{7}$ Department of Pedodontics and Preventive Dentistry, Rural Dental College, Pravara Institute of Medical Sciences, Loni Maharashtra, India

Corresponding Author: Anjum AJ Farooqui, Department of Oral Medicine and Radiology, Rural Dental College, Pravara Institute of Medical Sciences, Loni, Maharashtra, India, Phone: +919448759306, e-mail: fanjumj@gmail.com
}

compared with control group $(p<0.05)$. A significant difference was observed between precancerous and cancerous groups with GGT values at $5 \%$ level of significance. The mean GGT value is significantly higher in cancerous group as compared with precancerous group $(p<0.05)$.

Conclusion: There is a remarkable increase in salivary GGT activity in both precancerous and cancerous conditions. The increased activity was more marked in cancerous conditions than in precancerous conditions. The GGT levels were two- to threefold increased in precancerous conditions as compared with control group. This finding was statistically significant and also suggested the strong correlation between GGT levels and presence of precancerous conditions.

Clinical significance: Integration of all these observation strengthens our thinking that elevation of salivary GTT in precancerous and cancerous conditions can be a reliable biomarker in early detection and prevention of oral malignancy.

Keywords: Cancerous, Gamma-glutamyl transpeptidase, Precancerous, Tumor marker.

How to cite this article: Farooqui AAJ, Thete SG, Bangi S, Khalekar YJ, Nikumbh ND, Jadhav KB, Kathariya MD. Study to assess Activity and Concentration of Gamma-glutamyl Transpeptidase in Precancerous and Cancer Patients and Its Comparison with Controls. J Contemp Dent Pract 2017;18(10):943-946.

Source of support: Nil

Conflict of interest: None

\section{INTRODUCTION}

The word neoplasia means new growth. A neoplasm "is an abnormal mass of tissue, the growth of which exceeds and is uncoordinated with that of normal tissues, and which persists in the same excessive manner after cessation of the stimuli which evoked the change." ${ }^{1}$ The common term used for malignant tumors is cancer. The word cancer is derived from the Latin word for crab. 
The sixth most common malignancy in the world is oral carcinoma. It accounts for approximately $4 \%$ of all cancers and $2 \%$ of all cancer deaths worldwide. In India, it accounts for 50 to $70 \%$ of all cancers diagnosed. ${ }^{2}$

Early detection is necessary for the prompt treatment. Most commonly, laboratory diagnostic procedures are advised for early diagnosis. It involves the analysis of cellular and chemical constituents of blood. Various other biologic fluids are utilized for the diagnosis of disease. One of them is saliva. Saliva offers some distinctive advantages. Whole saliva can be collected noninvasively and by individuals with limited training. No special equipment is required for collection of the fluid. The noninvasive nature of collection, the direct contact to the oral tissue, and the relationship of enzymes between oral fluids and blood levels make saliva a useful and promising specimen. ${ }^{3}$

Tumor markers are biologic or biochemical substances produced by tumors cells and secreted into blood, other body fluids, or body tissues of some patients with certain types of cancer in higher than normal amounts. A tumor marker may be produced by the tumor itself, or by the body in response to the presence of cancer. ${ }^{1}$

Changes of enzyme activity in tissues, body fluids, and serum as well as changes of isozymes and enzyme variants in patients with malignancy suggest that these parameters may be used in the diagnosis of some cancers. ${ }^{4}$

Recently, GGT has been shown to increase considerably in the presence of various malignant tumors and precancerous lesions. This finding has drawn attention to GGT as a tumor marker. Of several enzymes, GGT is a common marker of human epithelial tumors, which mainly arises from epithelial layers. ${ }^{5}$

\section{MATERIALS AND METHODS}

Our study was aimed at assessing the activity of salivary GGT and its implication in the detection of oral malignancy. Patients reporting to the out patient department of our institute and cancer hospital, presenting with oral cancer, and precancerous conditions/lesions were selected and included in the study.

Individuals in the age group of above 20 years were included in the study. In this study, salivary GGT was analyzed in 75 cases. The selected patients were divided into three main groups as group I (controls with normal health), group II (patients with precancerous lesions and conditions), and group III (patients with oral cancer lesions).

Patients or controls medically compromised with diabetes and liver diseases were excluded from the study, after confirmation with history, clinical examination, and necessary laboratory investigations. Precancerous and cancerous lesions were confirmed with clinical diagnosis and histopathological examinations.
Whole saliva sample was collected from patients, preserved, and analyzed during the first 24 hours of collection. Whole saliva, which is secreted without any stimulation, was collected by making the patient spit or expectorate saliva into a sterile test tube. All the saliva samples were centrifuged at $3500 \mathrm{rpm}$ and dialyzed. The dialyzed samples were again centrifuged at $3500 \mathrm{rpm}$ for 15 minutes; the supernatant was used without concentration.

The GGT activity was assayed according to szasz methodology using gamma GT-kit. An enzyme solution was prepared by mixing 4 volumes of reagent 1 with 1 volume of reagent 2 and total working reagent was about $1000 \mu \mathrm{L}$. Sample of $100 \mu \mathrm{L}$ was taken, mixed, and incubated with working reagent at $37^{\circ} \mathrm{C}$ for 1 minute. The amount of 5-amino-2-nitrobenzoic acid realized was detected photochemically by reading the absorbance at $405 \mathrm{~nm}$.

Comparison of three groups (control, precancerous, and cancerous) with respect to GGT values was done by one-way analysis of variance (ANOVA).

Pairwise comparison of three groups (control, precancerous, and cancerous) with respect to GGT values was done by Newman-Keuls post hoc procedures.

\section{RESULTS}

Mean and standard deviation of GGT values in three groups (control, precancerous, and cancerous) (Table 1) are tabulated.

We have observed that there is a significant difference between three groups (control, precancerous, and cancerous) with respect to GGT values ( $\mathrm{F}=140.6235, \mathrm{p}<0.05)$ at $5 \%$ level of significance. It means that the mean GGT value is different in all the three groups (Table 2).

The mean GGT value is significantly higher in the precancerous group as compared with the control group $(p<0.05)$. A significant difference was observed between control and cancerous groups with GGT values at 5\% level of significance. The mean GGT value is significantly higher in cancerous group as compared with control group $(\mathrm{p}<0.05)$. A significant difference was observed between precancerous and cancerous groups with GGT values at $5 \%$ level of significance. The mean GGT value is significantly higher in cancerous group as compared with precancerous group $(\mathrm{p}<0.05)$ (Table 3).

Table 1: Mean and SD of GGT values in three groups (control, precancerous, and cancerous)

\begin{tabular}{llll}
\hline Groups & $N$ & Means \pm SD & Standard error \\
\hline Control & 25 & $24.75 \pm 9.94$ & 1.99 \\
Precancerous & 25 & $54.83 \pm 7.72$ & 1.54 \\
Cancerous & 25 & $80.53 \pm 16.04$ & 3.21 \\
\hline
\end{tabular}

SD: Standard deviation 
Study to assess Activity and Concentration of GGT in Precancerous and Cancer Patients

Table 2: Comparison of three groups (control, precancerous, and cancerous) with respect to GGT values by one-way ANOVA

\begin{tabular}{llllll}
\hline Source of variation & Degrees of freedom & Sum of squares & Mean sum of squares & F-value & $p$-value \\
\hline Between groups & 2 & 38970.72 & 19485.359 & 140.6235 & $0.0000^{*}$ \\
Within groups & 72 & 9976.61 & 138.564 & \\
\hline Total & 74 & 48947.33 & & \\
\hline
\end{tabular}

*Significant at 0.001; ANOVA: Analysis of variance

Table 3: Pairwise comparison of three groups (control, precancerous, and cancerous) with respect to GGT values by Newman-Keuls post hoc procedures

\begin{tabular}{llll}
\hline Groups & Control & Precancerous & Cancerous \\
\hline Mean & 24.7480 & 54.8330 & 80.5270 \\
Control & - & - & - \\
Precancerous & $\mathrm{p}=0.0001^{*}$ & - & - \\
Cancerous & $\mathrm{p}=0.0001^{*}$ & $\mathrm{p}=0.0001^{*}$ & - \\
\hline
\end{tabular}

*Significant at 0.001

\section{DISCUSSION}

The second most common cause of death is malignancy. The most common oral carcinomas are squamous cell carcinomas. It is the sixth most common malignancy. There are many advances in treatment modalities, which have led to improvements in the early detection of squamous cell carcinoma, but have not significantly impacted the overall survival rates of malignant patients (about $55 \%$ at 5 years). Therefore, it is necessary for detection of malignancy in the earliest possible changes, so as to improve overall survival rate. ${ }^{1}$

The GGT is present in various mammalian tissues and it is routinely studied from various tissues, such as kidney, brain, liver, and other tissues. It is also present in various body fluids, such as saliva, serum, and urine. ${ }^{6}$

The literature contains limited information on the salivary GGT except for a study done by Sajjan and Hinchigeri et al, ${ }^{7}$ who have detected and chromatographically separated GGT activity present in normal saliva and compared that of oral cancer patients.

Yao et $\mathrm{al}^{8}$ concluded in their study that GGT is one of the clinical values that is highly correlated with biotransformation, nucleic acid, metabolism and tumorigenesis, and is a sensitive biomarker reflecting cancer lesions.

Many researchers studied extensively the relation of GGT to hepatocarcinogenesis. Its fundamental cause is the activation of proto-oncogene or the inactivation of tumor suppressor genes initiated by carcinogens. $., 9,10$

A highly positive correlation can be found between total hepatic GGT expression and this abnormal regulation of genes leads to cancerization.

The main aim of our study was to determine the significance of salivary GGT in oral carcinoma and precancerous conditions. Although literature cited for salivary GGT is less compared with that of serum GGT in malignancy, interestingly the characteristics of salivary GGT were same as that of serum GGT.
Increased GGT activity has been demonstrated in various human carcinomas including oral squamous cell carcinoma by Fiala et al, ${ }_{11}^{11}$ Calderon-Solt and Solt,${ }^{6}$ and Mock et $\mathrm{al}^{5}$ and in experimentally induced premalignant and malignant lesions, as shown by Yao et al. ${ }^{12}$

In this study, variations in levels of salivary GGT in normal, precancerous, and cancerous conditions are studied.

It is clear from Table 3 that there is a remarkable increase in salivary GGT activity in both precancerous and cancerous conditions and increase was more marked in cancerous conditions than in precancerous conditions.

All the similar previous observations, coupled with our results, suggest that GGT activity is seen in carcinomas of oral region and can be a useful indicator in diagnosis and detection of oral cancer lesions. In the current study, we observed twofold increases in GGT level in precancerous group as compared with normal participants. This finding is statistically significant and also suggests the strong correlation between GGT and precancerous conditions.

The marked elevation of its activity proves that GGT activity is considered to be a useful aid for early diagnosis, predicting tumor extent and determining parameters of treatment efficacy and prognosis for oral cancer.

Since we studied the activity of GGT in the most common known cases of premalignant conditions, such as oral submucous fibrosis and premalignant lesions, such as leukoplakia, further subsequent analysis will define more clearly the role of GGT level as a diagnostic and prognostic indicator in patients with precancerous conditions and lesions. Hirata et a ${ }^{13}$ had observed similar increase in the GTT activity, though the study was done using plasma.

Integration of all these observation strengthens our thinking that elevation of salivary GTT in precancerous and cancerous conditions can be a reliable biomarker in early detection and prevention of oral malignancy.

\section{CONCLUSION}

Within the scope and limitations of the present study, the following conclusions can be drawn: there is a remarkable increase in salivary GGT activity in both precancerous and cancerous conditions. The increased activity was more marked in cancerous conditions than in precancerous conditions. Salivary GGT levels can be used as a reliable 
biomarker for early detection of malignant conditions. It can also be used as a noninvasive procedure for detection and timely referral of the patient to the oncologist. However, further studies on larger sample sizes are required to support our findings.

\section{REFERENCES}

1. Kumar, V.; Robbins, SL.; Cotran, RS. Pathologic basis of diseases. 5th ed. New York, Philadelphia (PA), Pennsylvania: Elsevier Publisher; 2004. p. 270, 272, 279, 280.

2. Park, K. Park's textbook of preventive and social medicine. 18th ed. Jabalpur: M/s Banarasidas Bhanot; 2005. p. 307.

3. Kaufman E, Lamster IB. The diagnostic applications of salivaa review. Int Am Assoc Dent Res 2002 Feb;13(2):197-212.

4. Chatterjee, MN.; Shinde, R. Text book of biochemistry. 7th ed. New Delhi: Jaypee Brothers Medical Publishers; 2008. p. 751.

5. Mock D, Whitestone B, Freeman J. Gamma-glutamyl transpeptidase activity in human oral squamous cell carcinoma. Oral Surg Oral Med Oral Pathol 1987 Aug;64(2):197-201.

6. Calderon-Solt L, Solt DB. Gamma-glutamyl transpeptidase in precancerous lesions and carcinomas of oral, pharyngeal, and laryngeal mucosa. Cancer 1985 Jul;56(1):138-143.

7. Sajjan AR, Hinchigeri SB, Datta KS. Multiple forms of gammaglutamyl transpeptidase in human submandibular gland. Clin Chem Acta 1991 Mar;197(2):133-139.
8. Yao DF, Dong ZZ, Yao DB, Wu XH, Wu W, Qiu LW, Wang HM, Meng XY. Abnormal expression of hepatomaderived gamma-glutamyl transferase subtyping and its early alteration for carcinogenesis of hepatocytes. Hepatobiliary Pancreat Dis Int 2004 Nov;3(4):564-570.

9. Jiménez-Alonso J, Jaimez L, Barrios L, Pérez-Jiménez F, García A, Bejarano R, Ogea JL, Jiménez-Pereperez JA. Gammaglutamyltransferase and alkaline phosphatase in the serum and saliva of patients with hepatic tumors and hepatic cirrhosis. Rev Esp Oncol 1984;31(2):315-319.

10. Tsutsumi M, Sakamuro D, Takada A, Zang SC, Furukawa T, Taniguchi N. Detection of a unique gamma-glutamyl transpeptidase messenger RNA species closely related to the development of hepatocellular carcinoma in humans: a new candidate for early diagnosis of hepatocellular carcinoma. Hepatology 1996 May;23(5):1093-1097.

11. Fiala S, Trout EC Jr, Teague CA, Fiala ES. Gammaglutamyltransferase, a common marker of human epithelial tumors? Cancer Detect Prev 1980;3(2):471-485.

12. Yao DF, Dong ZZ. Hepatoma-related gamma-glutamyl transferase in laboratory or clinical diagnosis of hepatocellular carcinoma. Hepatobiliary Pancreat Dis Int 2007 Feb;6(1): 9-11.

13. Hirata S, Odajima T, Kohama GI, Ishigaki S, Niitsu Y. Significance of glutathione S-transferase- $\pi$ as a tumor marker in patients with oral cancer. Cancer 1992 Nov;70(10): 2381-2387. 